\title{
Radial velocities, rotations, and duplicity of a sample of early F-type dwarfs ${ }^{\star}$
}

\author{
B. Nordström ${ }^{1,2}$, R.P. Stefanik ${ }^{2}$, D.W. Latham ${ }^{2}$, and J. Andersen ${ }^{1,2}$ \\ 1 Astronomical Observatory, Niels Bohr Institute for Astronomy, Physics \& Geophysics, Juliane Maries Vej 30 , DK - 2100 \\ Copenhagen, Denmark \\ ${ }^{2}$ Harvard-Smithsonian Center for Astrophysics, 60 Garden Street, Cambridge, MA 02138, U.S.A.
}

Received October 9, 1996; accepted February 17, 1997

\begin{abstract}
We present new radial and rotational velocities for 595 nearby early $\mathrm{F}$ dwarfs, based on digital spectra cross-correlated with individually optimised synthetic template spectra. The selection of optimum templates, the determination of rotational velocities, and the extraction of velocities from the blended spectra of double-lined spectroscopic binaries are discussed in some detail. We find 170 spectroscopic binaries in the sample and determine orbits for 18 double-lined and 2 single-lined binaries, including some spectroscopic triples. 73 stars are listed with too rapid rotation to yield useful radial velocities (i.e. $v \sin i>120 \mathrm{~km} \mathrm{~s}^{-1}$ ). We discuss the binary frequency in the sample, and the influence of unrecognised binaries on the definition of clean metallicity groups of young $\mathrm{F}$ dwarfs and the determination of their kinematical properties.
\end{abstract}

Key words: methods: statistical — techniques: radial velocities - stars: binaries: spectroscopic — stars: kinematics — Galaxy: solar neighbourhood

\section{Introduction}

Positions, velocities, metallicities, and ages of stars are the raw material from which samples of the stellar populations in the Milky Way galaxy are defined. Correlations between these parameters provide clues to the chemical and dynamical evolution of our home galaxy. Yet, after decades of observation and study, controversy remains concerning the true relationships between these basic observational

Send offprint requests to: B. Nordström (Danish address):

E-mail: birgitta@astro.ku.dk

* Tables 1, 5 and 6 are only available, and Tables 2-4 also available in electronic form at the CDS via anonymous ftp to cdsarc.u-strasbg.fr (130.79.128.5) or via http://cdsweb.ustrasbg.fr/Abstract.html parameters even for the solar neighbourhood, and even more so concerning their interpretation. Thus, as models of the formation and evolution of the galactic disk improve, the need for more definitive observational data becomes ever more pressing.

Coordinated efforts are being made towards this goal on two fronts: On the one hand, the chemical evolution of nearby solar-type stars has been studied in detail by Edvardsson et al. (1993; E93) using high-resolution spectroscopy. In parallel, a major programme has been undertaken to complete an inventory of the solar neighbourhood in order to define large, homogeneous, complete, and kinematically unbiased samples of nearby $F$ and $\mathrm{G}$ dwarf stars (see, e.g. Strömgren 1987; Nordström et al. 1996). Sample selection is based on the all-sky $u v b y-\mathrm{H} \beta$ photometry by Olsen $(1983,1993,1994 a, b)$, which allows the determination of distances, metallicities and individual ages. PPM proper motions (Bastian \& Röser 1991-1993) have been improved using modern positions observed with the Carlsberg Automatic Meridian Circle (Carlsberg 19911994). Finally, multiple radial-velocity observations have been obtained of the F and G dwarfs in the sample, mostly with the CORAVEL scanners (Mayor 1985). Altogether, complete data have now been collected for some 10000 stars in both hemispheres. The programme is further described in Nordström et al. (1996).

Different subgroups of these stars are interesting for a variety of purposes. At the old, metal-poor end of the sample, the delineation and characterisation of the old thin disk, thick disk, and halo populations are the main interest. On the other hand, the early $\mathrm{F}$ dwarfs for which reliable ages and metallicities can be determined, but which are too young to have moved far from their place of formation, are of prime importance in clarifying the cause(s) of the scatter in the local age-metallicity diagram (see Sect. 5). In all cases, radial velocities are needed in order to compute space motions and galactic orbits for individual stars. In addition, repeated radial-velocity observations allow the detection of spectroscopic binaries. This is 
important because large-amplitude binaries might bias the kinematical parameters derived for the samples of stars to which they belong. In addition, unrecognised binaries with significant amounts of secondary light of a colour different from that of the primary may be classified incorrectly when photometric age or metallicity indicators are used to subdivide the initial sample. Detecting such binary systems and analysing their composite spectra is the first step in correcting for these effects.

The class of early F-type dwarfs contains a large proportion of rapidly rotating stars $\left(v \sin i>40 \mathrm{~km} \mathrm{~s}^{-1}\right)$, for which radial velocities cannot be accurately determined with CORAVEL. We have therefore observed that part of our local sample with the Center for Astrophysics (CfA) Digital Speedometers (Latham 1985, 1992), which can handle rotations up to $\sim 120 \mathrm{~km} \mathrm{~s}^{-1}$ (Nordström et al. 1994; Paper I), in order to determine their radial and rotational velocities and search for binaries in the sample.

The present paper describes the selection of the sample and our observation and reduction procedures, gives results for 595 stars, including preliminary spectroscopic orbits for 18 double-lined and 2 single-lined binaries, and discusses the binary frequency in the sample and the extent to which such binaries may have been inadvertently included in the sample. Several forthcoming papers will further discuss the evolution of the galactic disk, based on the full data set (see, e.g. Nordström et al. 1997).

\section{Sample selection}

From the catalogue by Olsen (1983) of four-colour photometry of all A5-G0 stars in the HD catalogue to $m_{v}=$ 8.3, those $\mathrm{F}$ stars were selected which have evolved sufficiently off the ZAMS (i.e. by roughly $0.5-2.0 \mathrm{mag}$ in $M_{v}$ ) that individual ages can be determined from comparisons with theoretical isochrones. The following overall criteria were used (the precise $\delta c_{1}$ limit varying from 0.18 to 0.12 with $b-y$ within this range):

$$
\begin{aligned}
& 0.219<b-y<0.391 \\
& 0.066<\delta c_{1}<0.15
\end{aligned}
$$

Except that the selection of stars took place before $\mathrm{H} \beta$ photometry was complete for all the stars, these criteria are quite similar to those used by E93 to define their sample of stars.

As the CORAVEL observations got under way it was realized, however, that over half of the stars with $b-y<$ 0.27 were rotating too rapidly to yield usable radial velocities. The stars in the colour range $0.219<b-y<0.270$ and north of declination $-40^{\circ}$ were therefore transferred to the CfA instruments, together with a few later-type stars that were also rotating too rapidly for CORAVEL (and are found here to be short-period binaries). There is, however, enough overlap with the early CORAVEL results to ensure good consistency of the radial-velocity zero-points of the two systems.
Of the 669 stars on the original observing list (26\% of all stars in the Olsen catalogues in the same colour range and over the whole sky), results are presented here for a total of 595 stars (Tables 1 and 2), while 73 proved to be rotating too rapidly even for the CfA technique $(v \sin i>$ $120 \mathrm{~km} \mathrm{~s}^{-1}$ ); for completeness, these stars are listed in Table 4. A new, precise spectroscopic orbit for the doublelined eclipsing binary EI Cep, which is included in the photometrically defined sample, will be published separately.

\section{Observations and data reduction}

Echelle spectra for all programme stars were obtained with the 1.5-m Wyeth reflector at the Oak Ridge Observatory in Harvard, Massachusetts, the 1.5-m Tillinghast reflector on Mt. Hopkins, Arizona, and occasionally with the Multiple Mirror Telescope, also at Mt. Hopkins. Echelle spectra covering the range 5165.77 - $5211.25 \AA$ were obtained with the nearly identical CfA echelle spectrograph systems at all three telescopes and recorded with photoncounting intensified Reticon detectors. The instruments and standard observing and reduction procedures are described by Latham $(1985,1992)$. Standard observing procedures were followed throughout, but with increased exposure levels for the more rapidly-rotating stars which need better signal-to-noise in order to ensure reliable velocities.

For the determination of radial velocities from these spectra for stars with a wide range of rotations, we have developed a digital cross-correlation procedure based on the XCSAO task (Kurtz et al. 1992) as implemented in the IRAF environment ${ }^{1}$, using a large grid of synthetic template spectra covering the ranges in effective temperature, gravity, and rotation of our programme stars. The details of our technique and its performance have been documented in Paper I and will not be repeated here. We comment briefly below on its practical application to the programme stars, and on two refinements in the technique implemented since Paper I.

The grid of synthetic template spectra used here was computed for $[\mathrm{Fe} / \mathrm{H}]=0.0$, as the $m_{1}$ index for the programme stars indicated that they all have metallicities sufficiently close to solar. Effective temperatures range from 6000 to $7000 \mathrm{~K}$ in steps of $250 \mathrm{~K}, \log g$ from 4.5 to 3.0 in steps of $0.5 \mathrm{dex}$, and $v \sin i$ from 0 to $100 \mathrm{~km} \mathrm{~s}^{-1}$ in steps of $10 \mathrm{~km} \mathrm{~s}^{-1}$ and from 100 to $200 \mathrm{~km} \mathrm{~s}^{-1}$ in steps of $20 \mathrm{~km} \mathrm{~s}^{-1}$. Optimum templates were selected for the individual programme stars as follows:

For the stars with known $\mathrm{H} \beta$ indices and reddenings (about $3 / 4$ of the sample), Dr. E.H. Olsen kindly computed the individual $T_{\text {eff }}$ and $\log g$ values, using the Magain (1987) calibration for $T_{\text {eff }}$ and a procedure similar

1 IRAF is distributed by the National Optical Astronomy Observatories, which are operated by the Association of Universities for Research in Astronomy under cooperative agreement with the National Science Foundation. 
Table 2. Data for stars with double-lined or composite spectra. Coordinates are for equinox J2000; $V_{0}$ and $\mathrm{m} . e$. in $\mathrm{km} \mathrm{s}^{-1}$, and Span in days

\begin{tabular}{|c|c|c|c|c|c|c|c|c|c|c|c|c|}
\hline HD & $\mathrm{RA}$ & Dec & Temp1 & Temp2 & $\alpha$ & $n$ & $V_{0}$ & m.e. & Span & $q$ & m.e. & Rem. \\
\hline 5153 & $00: 53: 36.9$ & $+24: 55: 58$ & $7250 / 010$ & $7000 / 010$ & 0.65 & 3 & -24.61 & 0.29 & 780 & 0.926 & 0.015 & SB2W \\
\hline 5843 & 01:00:13.1 & $+11: 56: 07$ & $7000 / 010$ & $6750 / 010$ & 0.73 & 25 & -5.64 & 0.24 & 1471 & 0.921 & 0.018 & $\mathrm{SB} 2 \mathrm{O}$ \\
\hline 6469 & 01:06:08.4 & $+35: 30: 58$ & $7250 / 120$ & $6750 / 000$ & 0.06 & 6 & 12.76 & 2.66 & 1290 & & & SB2C \\
\hline 8026 & $01: 20: 47.7$ & $+56: 12: 28$ & $7250 / 120$ & $6750 / 000$ & 0.07 & 5 & -0.28 & 1.01 & 1809 & & & $\mathrm{SB} 2 \mathrm{C}$ \\
\hline 8136 & $01: 22: 39.8$ & $+68: 33: 55$ & $6750 / 010$ & $6750 / 010$ & 0.87 & 8 & -19.48 & 1.29 & 1128 & 1.101 & 0.045 & SB2W \\
\hline 10629 & $01: 44: 30.7$ & $+39: 57: 30$ & $7000 / 120$ & $7000 / 030$ & 0.41 & 3 & -4.08 & 1.20 & 1028 & & & SB2C \\
\hline $12180 \mathrm{~B}$ & 01:58:60.0 & $-22: 55: 03$ & $6500 / 000$ & $6500 / 000$ & 1.00 & 3 & 29.46 & 0.23 & 751 & 0.976 & 0.014 & SB2W \\
\hline 12637 & 02:04:45.8 & $+39: 26: 06$ & $7250 / 100$ & $6750 / 000$ & 0.11 & 11 & 6.13 & 1.00 & 1298 & & & SB2C \\
\hline 12984 & 02:07:01.8 & $-04: 13: 03$ & $6750 / 080$ & $6750 / 010$ & 0.15 & 3 & -14.56 & 6.89 & 753 & 0.952 & 0.665 & SB2W \\
\hline 14124 & $02: 19: 21.6$ & $+60: 29: 10$ & $7250 / 120$ & $6750 / 000$ & 0.39 & 5 & -1.16 & 0.72 & 1701 & & & SB2C \\
\hline 15227 & $02: 27: 32.0$ & $+16: 38: 36$ & $6750 / 000$ & $6750 / 000$ & 0.93 & 8 & 13.39 & 0.20 & 1446 & 0.978 & 0.019 & SB2W \\
\hline 17613B & $02: 49: 52.2$ & $+08: 56: 16$ & $6000 / 000$ & $5750 / 000$ & 0.37 & 6 & 2.26 & 0.40 & 820 & 0.875 & 0.062 & SB2W \\
\hline 19115 & 03:03:59.9 & $-17: 44: 16$ & $6750 / 030$ & $6500 / 030$ & 0.49 & 3 & 37.16 & 2.94 & 764 & 0.877 & 0.122 & SB2W \\
\hline 22054 & $03: 32: 13.1$ & $-31: 34: 13$ & $6750 / 000$ & $6500 / 000$ & 0.66 & 10 & 17.44 & 0.89 & 1090 & 0.914 & 0.037 & $\mathrm{SB} 2 \mathrm{O}$ \\
\hline 24623 & $03: 54: 34.9$ & $-09: 31: 13$ & $6750 / 000$ & $6500 / 000$ & 0.66 & 22 & 9.47 & 0.09 & 1452 & 0.982 & 0.004 & $\mathrm{SB} 2 \mathrm{O}$ \\
\hline 36845 & $05: 33: 43.5$ & $-18: 40: 42$ & $6750 / 100$ & $6500 / 000$ & 0.14 & 7 & 38.23 & 1.33 & 1741 & & & SB2C \\
\hline 37038 & $05: 35: 32.4$ & $-01: 50: 46$ & $7000 / 100$ & $6750 / 000$ & 0.23 & 9 & 17.06 & 0.38 & 1777 & & & SB2C \\
\hline $37603 \mathrm{~A}$ & $05: 40: 18.1$ & $+15: 21: 01$ & $7000 / 000$ & $6750 / 000$ & 0.80 & 10 & 21.01 & 0.73 & 1087 & 1.029 & 0.040 & SB2W \\
\hline 37603B & $05: 40: 18.1$ & $+15: 21: 01$ & $7000 / 000$ & $6750 / 000$ & 0.58 & 18 & 20.62 & & 1087 & 0.938 & 0.052 & $\mathrm{SB} 2 \mathrm{O}$ \\
\hline 39166 & 05:54:03.5 & $+57: 02: 44$ & $7000 / 000$ & $7000 / 000$ & 0.91 & 25 & 9.01 & 0.12 & 1800 & 0.997 & 0.006 & $\mathrm{SB} 2 \mathrm{O}$ \\
\hline 44849 & $06: 28: 21.9$ & $+62: 42: 44$ & $7250 / 100$ & $6750 / 010$ & 0.13 & 4 & 16.68 & 1.07 & 1044 & & & SB2C \\
\hline 54901 & $07: 11: 16.8$ & $+15: 19: 56$ & $7000 / 020$ & $6500 / 000$ & 0.17 & 24 & -9.86 & 0.15 & 1796 & 0.761 & 0 & $\mathrm{SB} 2 \mathrm{O}$ \\
\hline 57769 & $07: 24: 17.2$ & $+36: 18: 38$ & $6750 / 090$ & $6750 / 010$ & 0.09 & 11 & -34.31 & 3.08 & 1800 & & & SB2C \\
\hline 63424 & $07: 47: 10.1$ & $-39: 06: 12$ & $6750 / 000$ & $6750 / 000$ & 0.73 & 3 & -0.50 & 0.18 & 736 & & & SB2C \\
\hline 74425 & $08: 47: 50.8$ & $+66: 12: 38$ & $6000 / 050$ & $6000 / 040$ & 0.50 & 16 & 16.88 & 0.35 & 1321 & 0.857 & 0.00 & $\mathrm{SB} 2 \mathrm{O}$ \\
\hline 75638 & $08: 51: 26.3$ & $+12: 07: 32$ & $7000 / 080$ & $6750 / 010$ & 0.10 & 13 & 11.05 & 0.91 & 1346 & & & SB3O \\
\hline 76702 & 09:0 & $+81:$ & $7000 / 100$ & $6500 / 010$ & 0.0 & 6 & 6.38 & 1.32 & 1188 & & & SB2C \\
\hline 80853 & $09: 21: 10.3$ & $-35: 21: 25$ & $7000 / 100$ & $6750 / 010$ & 0.07 & 7 & 24.50 & 0.67 & 1211 & & & SB2C \\
\hline 85294 & 09:50:41.0 & $-07: 22: 56$ & $7000 / 090$ & $6750 / 010$ & $0.0^{\prime}$ & 9 & 28.36 & 1.70 & 1500 & 0.661 & 0.064 & SB2W \\
\hline 86579 & $09: 5$ ! & $-03: 04: 29$ & $7000 / 100$ & $6750 / 010$ & 0.11 & 7 & 7.26 & 1.42 & 1788 & 1.404 & 0.951 & SB2W \\
\hline 87810 & 10:07:07.6 & $-21: 15: 20$ & $6750 / 000$ & $6750 / 000$ & 1.00 & 23 & -0.24 & 0.07 & 1536 & 0.997 & 0.004 & $\mathrm{SB} 2 \mathrm{O}$ \\
\hline 88162 & $10: 10$ & $+20: 56: 43$ & $6750 / 010$ & 675 & 0.83 & 20 & -27.67 & 0.15 & 1774 & 0.952 & 0.022 & $\mathrm{SB} 2 \mathrm{O}$ \\
\hline 88788 & $10: 14: 19.5$ & $-12: 35: 03$ & $7000 / 120$ & $6500 / 010$ & 0.07 & 6 & 2.75 & 1.95 & 1479 & & & SB2C \\
\hline 92197 & $10: 38: 59.4$ & $+15: 58: 59$ & $6750 / 010$ & $6750 / 010$ & 0.80 & 6 & 8.88 & 2.11 & 1791 & 0.671 & 0.187 & SB2W \\
\hline 93785 & $10: 49: 15.0$ & $-26: 48: 55$ & $7000 / 120$ & $6750 / 030$ & 0.3 & 5 & 21.18 & 2.59 & 1502 & & & SB2C \\
\hline 94034 & $10: 50: 44.3$ & $-34: 29: 11$ & $6750 / 010$ & $6750 / 010$ & 0.91 & 5 & 6.03 & 0.26 & 797 & 1.098 & 0.051 & SB2W \\
\hline 96050 & $11 \cdot 04 \cdot 210$ & $-21: 07: 35$ & $6750 / 010$ & $6750 / 010$ & 0.87 & 4 & 6.31 & 1.28 & 1433 & 0.962 & 0.043 & SB2W \\
\hline 104636 & $12: 02: 59.9$ & $+41: 03: 55$ & $7000 / 100$ & $7000 / 000$ & 0.09 & 12 & 3.56 & 1.41 & 1216 & & & SB2C \\
\hline 108100 & & +42 : & $7000 / 080$ & & 0.17 & 7 & -7.76 & 1.04 & 1796 & & & SB2C \\
\hline 109557 & $12: 3$ & -16 : & $7000 /$ & $6750 / 020$ & 0.24 & 5 & 1.09 & 1.26 & 1765 & & & SB2C \\
\hline 110606 & $12: 43: 40.4$ & $-35: 24: 50$ & $7000 / 020$ & $7000 / 010$ & 0.81 & 5 & 4.74 & 0.28 & 790 & 0.912 & 0.034 & SB2W \\
\hline 121648 & $13: 56: 09.5$ & $+25: 55: 07$ & $6750 / 010$ & $6750 / 010$ & 0.86 & 30 & -29.50 & 0.27 & 1947 & 0.976 & 0.010 & $\mathrm{SB} 2 \mathrm{O}$ \\
\hline 128901 & $14: 37: 46.2$ & $+52: 01: 57$ & $7000 / 010$ & $7000 / 010$ & 0.75 & 7 & 7.69 & 7.42 & 1624 & & & SB2C \\
\hline 131773 & $14: 56: 21.6$ & $-29: 57: 36$ & $7000 /$ & & 0.1 & 4 & -9.88 & 0.69 & 830 & & & SB2C \\
\hline 134193 & $15: 0$ & $-11:$ & $6750 / 010$ & $6750 / 010$ & 0.5 & 13 & 4.77 & 0.22 & 1208 & 0.884 & 0.009 & $\mathrm{SB} 2 \mathrm{O}$ \\
\hline 135100 & $15: 11: 26.6$ & $+48: 41: 34$ & $6750 / 020$ & $6750 / 010$ & 0.67 & 6 & -14.84 & 0.18 & 1656 & 0.944 & 0.008 & SB2W \\
\hline 136276 & $15: 20: 09.3$ & $-13: 11: 30$ & $6750 / 010$ & $6750 / 010$ & 0.45 & 5 & -12.67 & 0.67 & 1209 & 0.828 & 0.024 & SB2W \\
\hline 147428 & $16: 20: 16.9$ & $+40: 15: 33$ & $6750 / 030$ & $6500 / 010$ & 0.18 & 19 & -19.66 & 0.18 & 1620 & 0.709 & 0.018 & $\mathrm{SB} 2 \mathrm{O}$ \\
\hline 155025 & $17: 07: 59.1$ & $+27: 06: 05$ & $7000 / 020$ & $7000 / 000$ & 0.46 & 8 & -16.78 & 1.55 & 1291 & 1.027 & 0.163 & SB2W \\
\hline 156458 & $17: 17: 36.9$ & & $6750 / 010$ & $6750 / 010$ & 0.39 & 15 & -30.65 & 0.18 & 1584 & 0.961 & 0.053 & $\mathrm{SB} 2 \mathrm{O}$ \\
\hline 159172 & $17: 33$ & $-17: 50: 10$ & $6500 / 010$ & $6500 / 000$ & 0.38 & 15 & 2.60 & 0.58 & 1484 & 0.8 & & $\mathrm{SB} 2 \mathrm{O}$ \\
\hline 171275 & $18: 32: 17.1$ & $+36: 25: 34$ & $7000 / 030$ & $7000 / 020$ & 0.29 & 28 & -30.87 & 0.18 & 1540 & 0.824 & 0.010 & $\mathrm{SB} 2 \mathrm{O}$ \\
\hline 176409 & & & $7000 / 100$ & & 0.0 & 8 & -28.30 & 1.57 & 1573 & & & SB2C \\
\hline 176613 & 19:02:19.6 & $-29: 20: 30$ & $7000 / 010$ & $7000 / 010$ & 0.90 & 4 & -1.76 & 0.32 & 1327 & 0.968 & 0.023 & SB2W \\
\hline
\end{tabular}

to that of Edvardsson et al. (1993) for $\log g$. The set of templates closest to these values was selected for each of these stars. For the remainder, we assumed $T_{\text {eff }}=7000 \mathrm{~K}$ and $\log g=4.0$ as our previous tests have shown these to be adequate (Paper I).

The optimum template rotation cannot be defined from the photometry, but must be found from test cor- relations of the observed spectra with the appropriate set of rotating templates. In Paper I, optimum template selection was based on the goodness-of-fit parameter $R$ (cf. Kurtz et al. 1992). As noted there, however, this criterion fails in the numerous cases of composite spectra. Considerable further experimentation led us to the conclusion that the maximum height of the correlation peak 
Table 2. continued

\begin{tabular}{|c|c|c|c|c|c|c|c|c|c|c|c|c|}
\hline HD & $\mathrm{RA}$ & Dec & Temp1 & Temp2 & $\alpha$ & $n$ & $V_{0}$ & m.e. & Span & $q$ & m.e. & Rem. \\
\hline 177005 & 19:01:14.7 & $+35: 37: 25$ & $7000 / 010$ & $7000 / 010$ & 0.19 & 13 & 2.79 & 0.49 & 1560 & 0.739 & 0.024 & $\mathrm{SB} 2 \mathrm{O}$ \\
\hline 192327 & $20: 14: 19.9$ & $+00: 08: 39$ & & $7000 / 000$ & & 8 & -32.85 & 0.49 & 1409 & & & SB2C \\
\hline 194032 & $20: 22: 20.4$ & $+29: 05: 26$ & & $7000 / 000$ & & 11 & -29.95 & 0.55 & 1596 & & & SB2C \\
\hline 195147 & $20: 10: 55.5$ & $+84: 32: 40$ & $7000 / 020$ & $7000 / 010$ & 0.50 & 4 & -11.81 & 0.89 & 1061 & 0.868 & 0.044 & SB2W \\
\hline 198226 & $20: 48: 06.2$ & $+27: 27: 24$ & $6750 / 030$ & $6750 / 040$ & 0.70 & 21 & -16.03 & 2.25 & 1471 & & & SB3O \\
\hline 203031 & 21:19:00.1 & $+26: 05: 23$ & $7000 / 050$ & $7000 / 010$ & 0.22 & 21 & 6.43 & 0.19 & 1474 & 0.677 & 0.015 & SB2O \\
\hline 205798 & $21: 36: 53.8$ & $+33: 43: 06$ & $7000 / 050$ & $7000 / 040$ & 0.25 & 10 & -25.47 & 1.10 & 1419 & 0.277 & 0.014 & SB2W \\
\hline 211244 & $22: 15: 26.6$ & $+18: 36: 11$ & $7000 / 070$ & $7000 / 060$ & 0.75 & 7 & 2.43 & 1.11 & 1386 & 0.895 & 0.025 & SB2W \\
\hline 215306 & $22: 43: 48.8$ & $+27: 41: 01$ & $7000 / 100$ & $7000 / 010$ & 0.25 & 7 & -11.66 & 0.55 & 1505 & & & SB2C \\
\hline 221431 & $23: 32: 18.4$ & $-33: 40: 32$ & $7000 / 020$ & $7000 / 000$ & 0.34 & 6 & -4.35 & 0.10 & 1377 & 0.898 & 0.005 & SB2W \\
\hline 222995 & $23: 45: 37.1$ & $+13: 09: 09$ & $6750 / 040$ & $6750 / 000$ & 0.30 & 19 & -20.28 & 0.25 & 1507 & 0.770 & 0.025 & $\mathrm{SB} 2 \mathrm{O}$ \\
\hline
\end{tabular}

Table 3. Orbital elements for spectroscopic binaries with orbital solutions. Mean errors are indicated below each parameter

\begin{tabular}{|c|c|c|c|c|c|c|c|c|}
\hline HD & $\begin{array}{l}P \\
{[\mathrm{~d}]} \\
\end{array}$ & $\begin{array}{c}T \\
\text { [HJD-2 } 440000] \\
\end{array}$ & $\begin{array}{c}V_{0} \\
{\left[\mathrm{~km} \mathrm{~s}^{-1}\right]}\end{array}$ & $\begin{array}{c}K_{1} \\
{\left[\mathrm{~km} \mathrm{~s}^{-1}\right]}\end{array}$ & $\begin{array}{c}K_{2} \\
{\left[\mathrm{~km} \mathrm{~s}^{-1}\right]}\end{array}$ & $e$ & $\begin{array}{c}\omega \\
{\left[{ }^{\circ}\right]} \\
\end{array}$ & $\begin{array}{r}\sigma_{1}, \sigma_{2} \\
{\left[\mathrm{~km} \mathrm{~s}^{-1}\right]}\end{array}$ \\
\hline \multirow[t]{2}{*}{005843} & 9.17607 & 7868.883 & -5.64 & 73.00 & 79.26 & 0.005 & 185.2 & 1.18 \\
\hline & 0.00016 & 1.792 & 0.24 & 0.33 & 1.35 & 0.004 & 70.4 & 4.96 \\
\hline \multirow[t]{2}{*}{022054} & 10.41302 & 7478.887 & 17.44 & 71.17 & 77.90 & 0.129 & 130.6 & 5.89 \\
\hline & 0.00038 & 0.146 & 0.89 & 2.40 & 0.78 & 0.010 & 4.3 & 0.91 \\
\hline \multirow[t]{2}{*}{024623} & 19.66227 & 7861.470 & 9.47 & 63.92 & 65.08 & 0.491 & 130.0 & 0.53 \\
\hline & 0.00037 & 0.014 & 0.09 & 0.19 & 0.22 & 0.002 & 0.3 & 0.64 \\
\hline \multirow[t]{2}{*}{ 037603B } & 27.76205 & 7886.104 & 20.62 & 20.43 & 21.79 & 0.405 & 349.6 & 2.71 \\
\hline & 0.00621 & 0.226 & 0.38 & 0.88 & 0.66 & 0.024 & 4.1 & 1.95 \\
\hline \multirow{2}{*}{039166} & 15.86860 & 7568.472 & 9.01 & 61.97 & 62.16 & 0.232 & 304.3 & 0.75 \\
\hline & 0.00037 & 0.034 & 0.12 & 0.26 & 0.27 & 0.003 & 0.8 & 0.80 \\
\hline \multirow[t]{2}{*}{054901} & 41.00333 & 7709.088 & -9.86 & 44.46 & 58.45 & 0.638 & 349.3 & 0.72 \\
\hline & 0.00125 & 0.024 & 0.15 & 0.26 & 0.72 & 0.004 & 0.5 & 2.36 \\
\hline \multirow[t]{2}{*}{074425} & 1.585232 & 7797.961 & 16.88 & 101.93 & 118.97 & 0.011 & 184.0 & 1.77 \\
\hline & 0.000008 & 0.100 & 0.35 & 0.64 & 0.79 & 0.005 & 22.6 & 2.19 \\
\hline \multirow[t]{2}{*}{075638} & 5.81667 & 7558.279 & 11.05 & 28.92 & & 0.083 & 322.0 & 2.96 \\
\hline & 0.00086 & 0.452 & 0.91 & 1.20 & & 0.056 & 28.2 & \\
\hline \multirow[t]{2}{*}{087810} & 12.94724 & 7442.274 & -0.24 & 55.50 & 55.68 & 0.439 & 48.6 & 0.47 \\
\hline & 0.00012 & 0.008 & 0.07 & 0.17 & 0.18 & 0.002 & 0.3 & 0.49 \\
\hline \multirow{2}{*}{088162} & 24.74771 & 7764.323 & -27.67 & 23.85 & 25.05 & 0.603 & 17.4 & 0.78 \\
\hline & 0.00310 & 0.038 & 0.15 & 0.32 & 0.44 & 0.009 & 0.93 & 1.10 \\
\hline \multirow[t]{2}{*}{121648} & 4.99167 & 7750.057 & -29.50 & 89.85 & 92.02 & 0.007 & 219.4 & 1.86 \\
\hline & 0.00003 & 0.512 & 0.27 & 0.56 & 0.72 & 0.004 & 36.9 & 2.39 \\
\hline \multirow[t]{2}{*}{134193} & 5.76930 & 7572.411 & 4.77 & 77.40 & 87.61 & 0.004 & 73.7 & 1.14 \\
\hline & 0.00006 & 0.930 & 0.22 & 0.50 & 0.48 & 0.005 & 57.9 & 1.07 \\
\hline \multirow[t]{2}{*}{147428} & 75.55870 & 7784.165 & -19.66 & 27.28 & 38.46 & 0.089 & 186.7 & 0.70 \\
\hline & 0.02198 & 1.059 & 0.18 & 0.25 & 0.83 & 0.014 & 5.3 & 2.71 \\
\hline \multirow[t]{2}{*}{156458} & 17.11603 & 7990.970 & -30.65 & 60.58 & 63.07 & 0.161 & 279.1 & 0.54 \\
\hline & 0.00030 & 0.078 & 0.18 & 0.24 & 3.06 & 0.004 & 1.7 & 8.74 \\
\hline \multirow{2}{*}{159172} & 13.87687 & 7674.910 & 2.60 & 54.89 & 66.78 & 0.218 & 204.1 & 3.61 \\
\hline & 0.00033 & 0.055 & 0.58 & 1.49 & 0.37 & 0.010 & 1.8 & 0.75 \\
\hline \multirow[t]{2}{*}{171275} & 15.40365 & 7839.525 & -30.87 & 58.78 & 71.37 & 0.083 & 305.4 & 0.91 \\
\hline & 0.00058 & 0.116 & 0.18 & 0.23 & 0.73 & 0.005 & 2.9 & 2.91 \\
\hline \multirow[t]{2}{*}{177005} & 11.10549 & 7943.389 & 2.79 & 53.24 & 72.04 & 0.333 & 143.3 & 2.86 \\
\hline & 0.00063 & 0.078 & 0.49 & 1.52 & 1.53 & 0.011 & 2.6 & 1.93 \\
\hline \multirow[t]{2}{*}{198226} & 1.49941 & 7773.106 & -16.03 & 90.51 & & 0.021 & 93.7 & 10.1 \\
\hline & 0.00004 & 0.421 & 2.25 & 3.27 & & 0.034 & 101.3 & \\
\hline \multirow{2}{*}{203031} & 48.73828 & 7760.086 & 6.43 & 27.49 & 40.59 & 0.493 & 159.0 & 1.29 \\
\hline & 0.00442 & 0.080 & 0.19 & 0.53 & 0.55 & 0.009 & 1.0 & 1.11 \\
\hline \multirow[t]{2}{*}{222995} & 85.83454 & 7819.892 & -20.28 & 31.73 & 41.20 & 0.335 & 175.0 & 2.17 \\
\hline & 0.02771 & 0.392 & 0.25 & 0.86 & 0.51 & 0.008 & 1.8 & 1.18 \\
\hline
\end{tabular}


is a more reliable criterion for choosing the rotating template which gives the best match to the observed spectra, and this method was adopted for the final correlations of all programme stars.

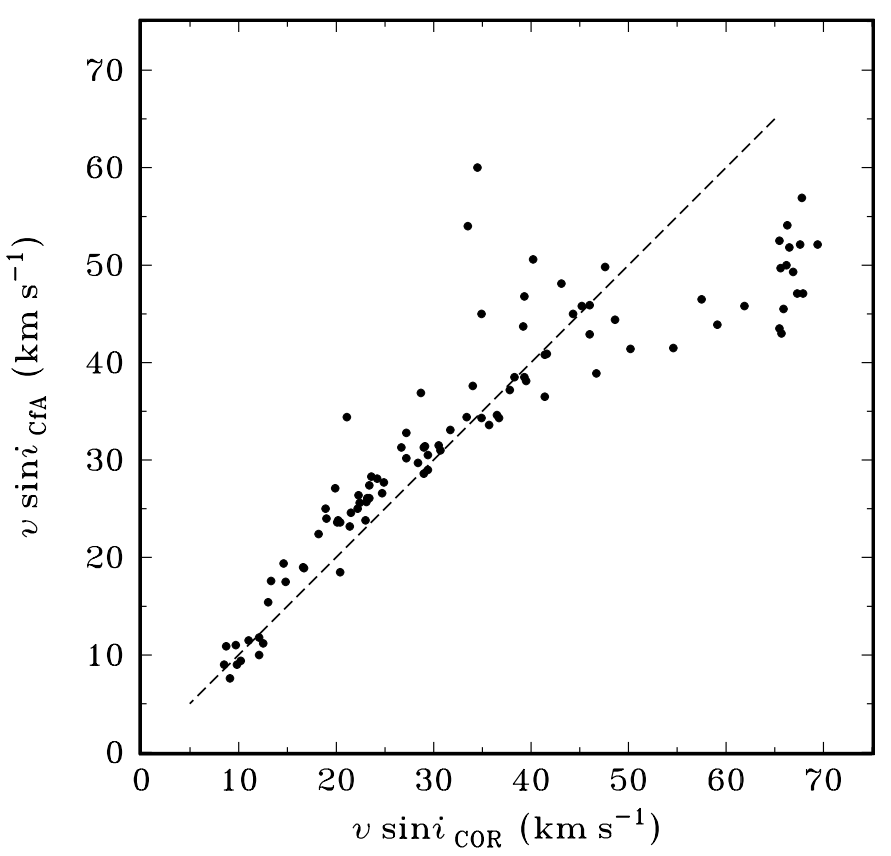

Fig. 1. Rotational velocities $\left(\mathrm{km} \mathrm{s}^{-1}\right)$ derived here vs. those observed with CORAVEL

From the correlation peak height as a function of template rotation, the value corresponding to maximum correlation height was estimated by quadratic interpolation. As shown in Fig. 1, this procedure leads to much better agreement with the CORAVEL rotations measured for the overlap of slowly-rotating stars common to the two programmes which was discussed in Paper I. The overall zero-point and scale differences in $v \sin i$ seen with the older procedure have disappeared, although there still seems to be a difference of $\sim 3 \mathrm{~km} \mathrm{~s}^{-1}$ for rotations around $20 \mathrm{~km} \mathrm{~s}^{-1}$. Given the uncertainties in the treatment of the instrumental profile and scattered light of our data and the simplifying assumptions underlying the calibration of both methods, the origin of this difference is not clear, but it remains within the fundamental uncertainty of $\sim 10 \%$ derived by Collins \& Truax (1995). The increased scatter seen for $v \sin i>40 \mathrm{~km} \mathrm{~s}^{-1}$ is presumably due to the rapidly deteriorating accuracy of the CORAVEL rotations for such broad-lined stars, due to the fixed CORAVEL mask which is optimised for sharp-lined stars.

We have verified that our radial-velocity zero-point, which was the key subject of Paper I, remains the same to within $100 \mathrm{~m} \mathrm{~s}^{-1}$ when using this modified procedure. Thus, our previous results on the systematic and random errors of our radial velocities remain valid for the data presented here.
The second refinement in our technique was the use of the two-dimensional cross-correlation algorithm TODCOR (Zucker \& Mazeh 1994), as implemented at CfA by $\mathrm{G}$. Torres, to extract radial velocities of both components from the observed, blended spectra of 65 doublelined spectroscopic binaries in our sample. For 18 of these systems we had enough velocities to allow solutions for double-lined orbits, and in another 21 cases the available pairs of primary and secondary velocities spanned a sufficient velocity range that we could use the Wilson (1941) method to derive systemic velocities and mass ratios.

The ability of TODCOR to extract reliable velocities even from strongly blended correlation peaks is quite remarkable, if a suitably fine grid of rotating templates is available. The absence of systematic velocity errors has been checked in a system with equal components by Latham et al. (1996). As an application of particular value in the present programme, we have been able to derive velocities with TODCOR for a considerable number of systems composed of rapidly-rotating late A-type primaries and sharp-lined late-type secondaries contributing only a few percent of the total light. For these systems the standard correlations against slowly-rotating templates only picked up the velocity of the slowly-rotating secondary. With TODCOR we were able to identify the spectrum of the rapidly-rotating primary and to solve for the velocities of both stars.

TODCOR can also provide an estimate of the light ratio $L_{2} / L_{1}$ between the two stars at the wavelength observed. We list this information when available, but have not performed a similarly exhaustive study of its accuracy as for the radial velocities, including, e.g., template optimization for the individual binary components.

\section{Results}

Table 1 (available in electronic form only; see footnote to title page) gives our main results for the 530 single-lined stars for which reliable radial velocities could be obtained (all velocity data in $\mathrm{km} \mathrm{s}^{-1}$ ).

In Table 1, Cols. 1-3 give HD numbers and J2000 coordinates, Col. 4 indicates the atmospheric parameters of the adopted synthetic template spectrum ( $t$ followed by the temperature and $g$ by $10 \times \log g$ ), and Col. 5 the rotational velocity of the star determined as described above.

Columns 6 and 7 give the number of observations, $n$, and the time span (days) between the first and last observation.

Columns 8-13 list, respectively, the mean radial velocity and its standard error, the standard deviation of the individual velocities, the average uncertainty of a single observation as computed by XCSAO (see Paper I), the ratio of observed to expected rms error, the observed $\chi^{2}$ and the probability $P\left(\chi^{2}\right)$ that it is due to the random errors of $n$ observations of an otherwise constant star. If the observed standard deviation is less than predicted by 
XCSAO (which often happens by chance when small numbers of observations are involved), the error of the mean velocity is given as the latter divided by $\sqrt{n}$. Columns 14 and 15 list, for reference, the average values of the goodness-of-fit statistic $\langle R>$ (Kurtz et al. 1992) and the height of the correlation peak.

Results for the 65 double-lined systems treated with TODCOR are given in Table 2. The first three columns are as in Table 1, while Cols. 4 and 5 give the parameters of the two templates (i.e. temperature and rotation; $\log g=4.0$ in all cases). Column 6 lists the mean luminosity parameter $\alpha=L_{2} / L_{1}$ provided by TODCOR, and Col. 7 the number of observations. The systemic velocity and its mean error are given in Cols. 8-9, derived, in order of preference, from a full orbital solution, from the available pairs of primary and secondary velocities using the Wilson (1941) method (in which the systemic velocity and mass ratio are derived from a fit of $V_{2}$ vs. $V_{1}$, assuming only momentum conservation), or as a straight mean. For systems with an orbital solution or analysed by the Wilson method, the mass ratio $(q)$ and its mean error are given in the following two columns. The remarks in the final column indicate double-lined (SB2) or triple (SB3) systems with either no significant velocity variation observed $(\mathrm{C})$, analysed by the Wilson method (W), or with an orbital solution $(\mathrm{O})$. For the triple systems, only a single-lined orbit for the short-period pair has been determined.

Table 4. Stars which rotate too rapidly for usable radial-velocity measurements with the CfA instruments

\begin{tabular}{clrlc}
\hline HD & HD & HD & HD & HD \\
\hline 4086 & 27832 & 81725 & 125488 & 181656 \\
4856 & 30315 & 84667 & 127457 & 182246 \\
6696 & 36844 & 88829 & 130353 & 190004 \\
7138 & 38610 & 89866 & 130667 & 196386 \\
8302 & 38753 & 91728 & 131160 & 198028 \\
8607 & $42313 \mathrm{BC}$ & 94206 & 131721 & 198047 \\
11284 & 46318 & 99946 & 135282 & 198346 \\
13179 & 51832 & 103846 & 141652 & 203405 \\
16453 & 54269 & 104622 & 142124 & 212048 \\
$17613 \mathrm{~A}$ & 63795 & 110220 & 145873 & 213619 \\
18570 & 72984 & 110456 & $150340 \mathrm{~A}$ & 217855 \\
19759 & 74781 & 111005 & 166600 & 218010 \\
23290 & 77214 & 118703 & 168066 & 219712 \\
26757 & 79725 & 119603 & 179280 & \\
27743 & 80678 & 121136 & 180005 & \\
\hline
\end{tabular}

The elements of the 18 double-lined and 2 single-lined spectroscopic orbits derived here are given in Table 3 , and the orbits are shown in Fig. 2. The 73 stars rotating more rapidly than $120 \mathrm{~km} \mathrm{~s}^{-1}$ and for which no velocities could be obtained are listed in Table 4 (HD numbers only).
Finally, the individual radial-velocity observations for single-lined and double-lined stars are listed in Tables 5 and 6 , respectively (provided in electronic form only).

\section{Discussion}

The immediate result of this paper is the catalogue of 595 mean radial velocities and derived quantities contained in Tables 1 and 2. As shown in Fig. 3, the typical mean error of the mean velocity of a constant star is $\sim 1 \mathrm{~km} \mathrm{~s}^{-1}$ for stars rotating less than $\sim 120 \mathrm{~km} \mathrm{~s}^{-1}$.

As our criterion for identifying spectroscopic binaries in the sample we adopt the limit $P\left(\chi^{2}\right) \leqq 0.01$ for significant radial-velocity variability, i.e. a confidence level of $99 \%$. The distribution of observed $P\left(\chi^{2}\right)$ values is displayed in Fig. 4 and shows a prominent peak of 170 stars in the bin $0.00-0.01$ in $P\left(\chi^{2}\right)$, corresponding to the spectroscopic binaries. It is also noteworthy, however, that the $P\left(\chi^{2}\right)$ distribution remains essentially flat in the entire range $0.01-1.00$, indicating that the internal error estimates from XCSAO which were used to compute $\chi^{2}$ are indeed realistic. If one relaxes the variability criterion to $P\left(\chi^{2}\right) \leqq 0.04$, the number of stars satisfying this criterion increases to 199 .

The bins $0.00-0.04$ in Fig. 4 still contain a random component of constant stars, corresponding to the average level in the rest of the $P\left(\chi^{2}\right)$ range, 4.1 stars per bin of 0.01 in $P\left(\chi^{2}\right)$. Hence, the 170 stars in the first bin should be reduced to 166 , and the 29 stars in the next three bins to 17 , giving a range for the true number of detected binaries of $166-182$, with a sampling error of $\sim 13$. The observed binary frequency is thus $29 \pm 2 \%$.

The raw binary fraction derived above must be corrected for the fact that our sample is limited by apparent magnitude, thus favouring the inclusion of binaries which are, on average, more luminous than the single stars. Suppose, as an example, that our double-lined binaries (Table 2) were on average $50 \%$ more luminous than single stars of the same colour. We would then include them from a volume that is a factor 1.84 larger than that in which the single stars are complete. Under this assumption, 30 of these stars would be removed from the sample for this reason, reducing the true frequency of detected binaries to $24 \pm 2 \%$. A similar effect will operate on the $\sim 100$ single-lined systems since the light of the secondaries will be present at some level, but we estimate that $\sim 10 \%$ is a plausible rough upper limit to their contribution. This suggests that fewer than $\sim 15$ single-lined binaries have been included from outside our completeness limit for single stars, reducing our estimate of the true frequency of detected binaries to perhaps $22 \pm 2 \%$.

A similar analysis of the $P\left(\chi^{2}\right)$ distribution of the Duquennoy \& Mayor (1991) volume-limited sample of G dwarfs (their Fig. 3) yields a fraction of radial-velocity variables of $\sim 37 \%$. If the true frequency of spectroscopic binaries is the same in the two samples, these figures 

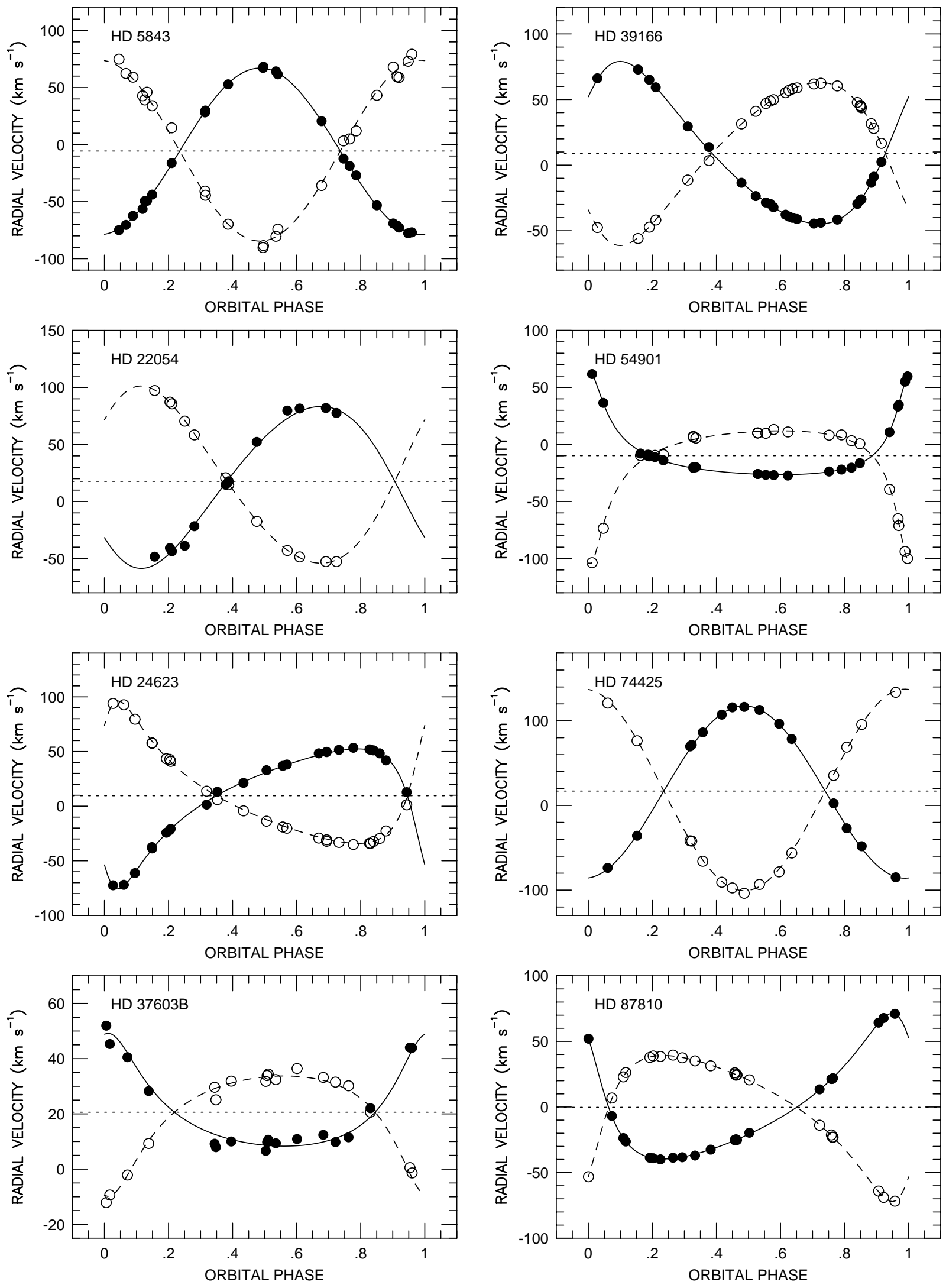

Fig. 2. Spectroscopic orbits and observed radial velocities for 20 binary systems. The orbital elements are given in Table 3 

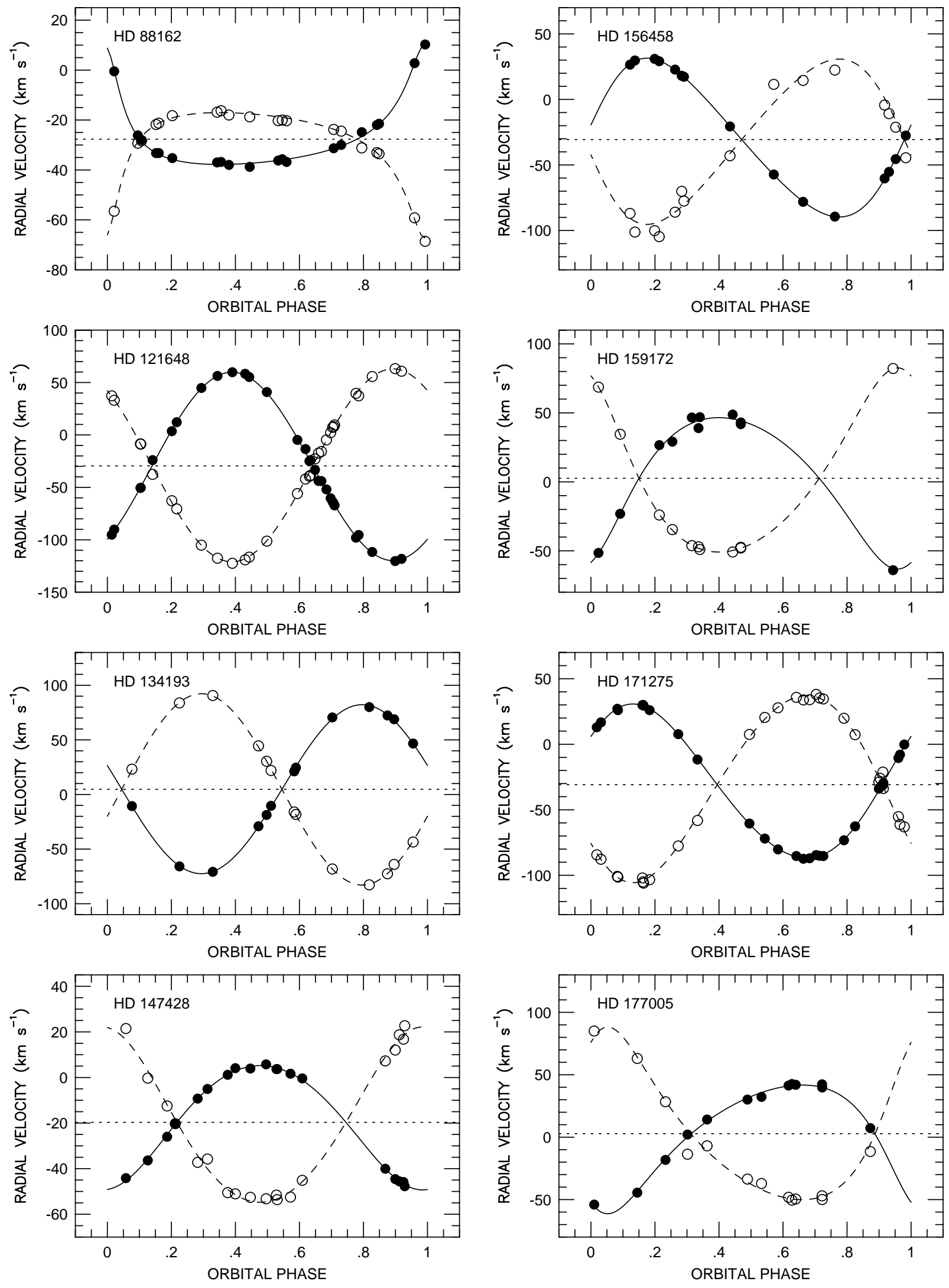

Fig. 2. continued 

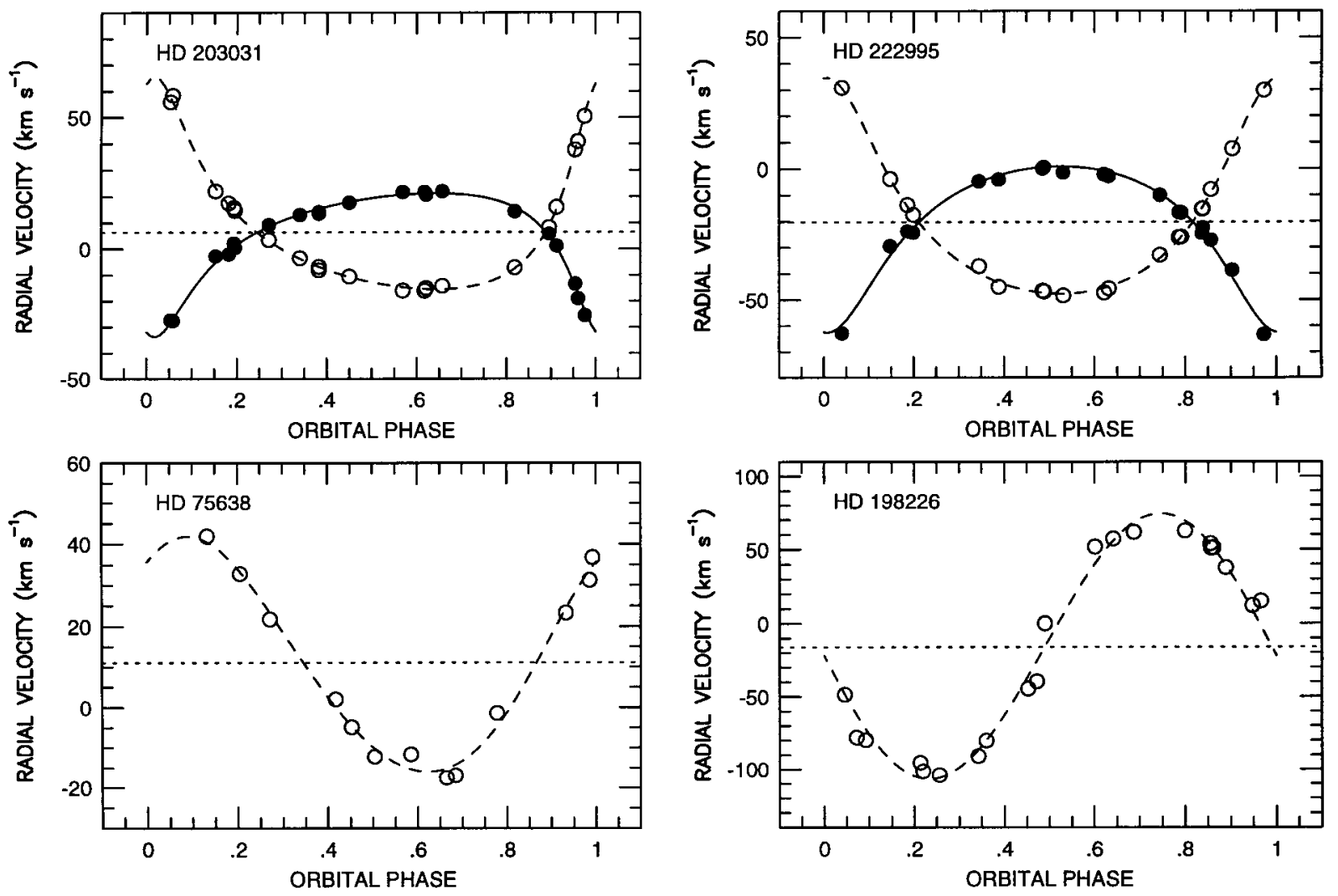

Fig. 2. continued

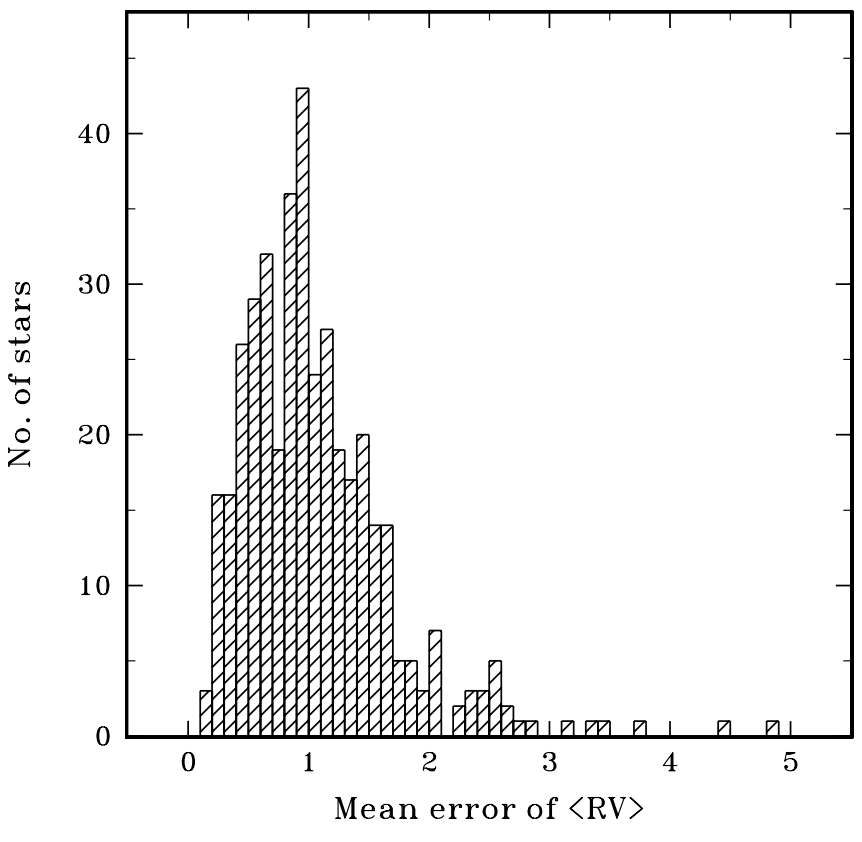

Fig. 3. Distribution of mean errors $\left(\mathrm{km} \mathrm{s}^{-1}\right)$ of the mean velocities of the 426 constant stars from Table 1 (defined by the criterion $P\left(\chi^{2}\right) \geqq 0.01$

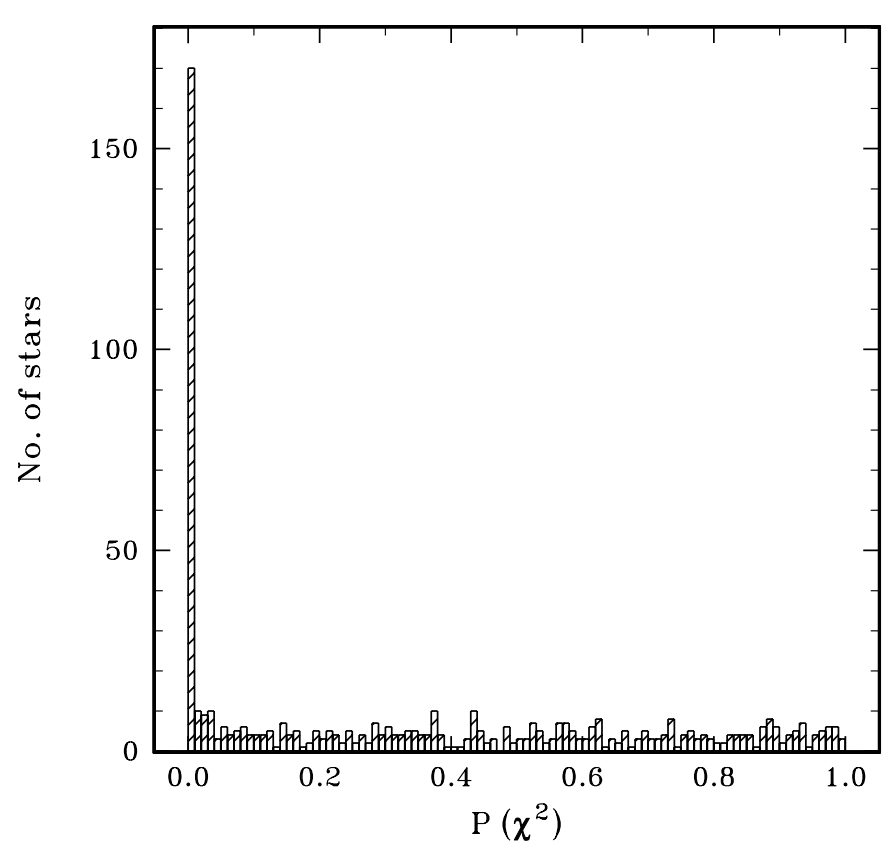

Fig. 4. Distribution of observed $P\left(\chi^{2}\right)$ values for all stars in Tables 1 and 2 with more than one observation (bin size 0.01 ) 
suggest that we have missed about half of the total number of spectroscopic binaries in our own sample. This is to be expected because the detection limit for velocity variability is significantly higher for our fast-rotating $\mathrm{F}$ stars, and the time span and number of observations per star are rather lower than in the study by Duquennoy \& Mayor.

The 24 double-lined systems consisting of a rapidlyrotating late A star and a sharp-lined cooler secondary are of special interest. As their combined uvby indices mimic those of the youngest, early F-type dwarfs, these stars would have appeared in that age group if their binary nature had gone unnoticed. Their derived metallicity would also have been affected by their composite nature, biasing the resulting age-metallicity diagram (AMD).

It has recently been proposed (Wielen et al. 1996) that the scatter in the AMD is the result of diffusion of disk star orbits. According to their model, the true birthplace of a star can be derived from its position in the AMD under the assumption of a single-valued local age-metallicity relation (AMR) and a radial abundance gradient in the disk: The farther a star is off the mean relation, the farther from the solar circle was it supposedly born. As a specific example, since the $[\mathrm{Fe} / \mathrm{H}]$ of the Sun is 0.17 dex higher than the average for nearby $\mathrm{F}$ dwarfs of the same age, it would supposedly have been formed at a galactocentric distance of $6.6 \mathrm{kpc}$. According to this theory, its orbit would then have been modified by interactions with massive objects in the disk (perhaps giant molecular clouds or massive black holes) so that it reached its present mean distance at $R_{0}=8.5 \mathrm{kpc}$ after $4.6 \mathrm{Gyr}$, and with its orbital eccentricity modified again to near its presumably small initial value.

When using $\mathrm{F}$ stars to test such an hypothesis (see Nordström et al. 1997), early F stars with high or low abundances are particularly important since they have had the least time to diffuse into the solar neighbourhood from their putative distant birthplaces. Even a few spurious points in the AMD due to unrecognised binaries of the type discussed above could lead to erroneous conclusions. The data presented here will help to avoid such mistakes.

Acknowledgements. The grid of synthetic template spectra computed by Jon Morse, with the model atmosphere programmes and spectral line lists by Robert Kurucz, were key elements in the work presented here, as were the XCSAO package and TODCOR implementations by our CfA colleagues. Financial support to BN and JA from the Carlsberg Foundation, the Danish Natural Science Research Council, and the Smithsonian Institution is gratefully acknowledged.

\section{References}

Bastian U., Röser S., 1991-93, PPM Star Catalogues, Vols. IIV, Astron. Rechen-Institut Heidelberg, Germany

Carlsberg Meridian Catalogue La Palma 1991-94, Observations of positions of stars and planets, Vols. 5-8. Copenhagen University Observatory, Royal Greenwich Observatory and Real Instituto y Observatorio de la Armada en San Fernando

Collins G.W. II, Truax R.J., 1995, ApJ 439, 860

Duquennoy A., Mayor M., 1991, A\&A 248, 485

Edvardsson B., Andersen J., Gustafsson B., Lambert D.L., Nissen P.E., Tomkin J., 1993, A\&A 275, 101 (E93)

Kurtz M.J., Mink D.J., Wyatt W.F., 1992, in: Worrall DM., Biemesderfer C., Barnes J. (eds.) Astronomical Data Analysis Software and Systems 1, ASPC 25, 432

Latham D.W., 1985, in: Philip A.G.D., Latham D.W. (eds.) Stellar Radial Velocities. L. Davis Press, Schenectady, N.Y., p. 21

Latham D.W., 1992, in: McAlister H., Hartkopf W. (eds.) Complementary Approaches to Binary and Multiple Star Research, IAU Colloq. 135, ASPC 32, 110

Latham D.W., Nordström B., Andersen J., et al., 1996, A\&A 314,864

Magain P., 1987, A\&A 187, 323

Mayor M., 1985, in: Philip A.G.D., Latham D.W. (eds.) Stellar Radial Velocities. L. Davis Press, Schenectady, N.Y., p. 35

Nordström B., Andersen J., Olsen E.H., et al., 1997, A\&A (submitted)

Nordström B., Latham D.W., Morse J., et al., 1994, A\&A 287, 338 (Paper I)

Nordström B., Olsen E.H., Andersen J., et al., 1996, in Burkert A., Hartmann D., Majewski S. (eds.) The History of the Milky Way and its Satellite System, ASPC 112, 145

Olsen E.H., 1983, A\&AS 54, 55

Olsen E.H., 1993, A\&AS 102, 89

Olsen E.H., 1994a, A\&AS 104, 429

Olsen E.H., 1994b, A\&AS 106, 257

Strömgren B., 1987, in: Gilmore G., Carswell R.F. (eds.) The Milky Way Galaxy. Reidel, Dordrecht, p. 299

Wielen, R., Fuchs, D., Dettbarn, C., 1996, A\&A 314, 438

Wilson O.C., 1941, ApJ 93, 29

Zucker S., Mazeh T., 1994, ApJ 420, 806 